\title{
THE ACTION OF THE STEENROD SQUARES ON THE MODULAR INVARIANTS OF LINEAR GROUPS
}

\author{
NGUYÊN H. V. HUNG
}

(Communicated by Frederick R. Cohen)

Dedicated to Professor Frank Peterson on his sixtieth birthday

\begin{abstract}
We compute the action of the Steenrod squares on the Dickson invariants of the group $G L_{n}=G L(n, \mathbf{Z} / 2)$ and the Mùi invariants of the subgroup $T_{n}$ consisting of all upper triangular matrices with 1 on the main diagonal. Our method is very elementary. Roughly speaking, we read off the above action from the expansion of the Mùi invariants in terms of Dickson and Mùi invariants of fewer variables.
\end{abstract}

\section{INTRODUCTION}

Let $G L_{n}=G L(n, \mathrm{Z} / 2)$ and let $T_{n}$ be the Sylow 2-subgroup of $G L_{n}$ consisting of all upper triangular matrices with 1 on the main diagonal. These two groups act on $\mathbf{Z} / 2\left[y_{1}, \ldots, y_{n}\right]$ in the usual manner.

Dickson and Mùi, respectively, computed the invariant rings

$$
\mathbf{Z} / 2\left[y_{1}, \ldots, y_{n}\right]^{G L_{n}} \text { and } \mathbf{Z} / 2\left[y_{1}, \ldots, y_{n}\right]^{T_{n}}
$$

respectively, as follows.

In [3] Mùi defined the invariant $V_{n}$ by

$$
V_{n}=V_{n}\left(y_{1}, \ldots, y_{n}\right)=\prod_{\lambda_{i} \in \mathbf{Z} / 2}\left(\lambda_{1} y_{1}+\cdots+\lambda_{n-1} y_{n-1}+y_{n}\right) \text {. }
$$

The Dickson invariant $Q_{n, s}=Q_{n, s}\left(y_{1}, \ldots, y_{n}\right)$, for $0 \leq s<n$, can be defined by the inductive formula

$$
Q_{n, s}=Q_{n-1, s-1}^{2}+V_{n} Q_{n-1, s} .
$$

Here, by convention, $Q_{n, n}=1$ for any $n$, and $Q_{1,0}=y_{1}$.

Dickson proved in [2] that

$$
\mathbf{Z} / 2\left[y_{1}, \ldots, y_{n}\right]^{G L_{n}}=\mathbf{Z} / 2\left[Q_{n, 0}, \ldots, Q_{n, n-1}\right] .
$$

Received by the editors April 5, 1990; the contents of this paper were presented in November 1989 to a seminar at MSRI, Berkeley.

1980 Mathematics Subject Classification (1985 Revision). Primary 55S05.

Key words and phrases. Modular invariants, Steenrod operations.

Research at MSRI supported in part by NSF Grant DMS-8505550. 
Mùi showed in [3] that

$$
\mathbf{Z} / 2\left[y_{1}, \ldots, y_{n}\right]^{T_{n}}=\mathbf{Z} / 2\left[V_{1}, \ldots, V_{n}\right]
$$

Let us consider the elementary abelian 2-group $E^{n} \cong(\mathrm{Z} / 2)^{n}$. As is well known $H^{*}\left(B E^{n}\right) \cong \mathrm{Z} / 2\left[y_{1}, \ldots, y_{n}\right]$ with $\operatorname{deg} y_{i}=1$. Here and in what follows, the cohomology is always taken with coefficients in $Z / 2$. The Dickson and Mùi invariants have successfully been used in algebraic topology by many authors since Mùi's work [3], where he proved, among other things, that

$$
\begin{aligned}
\mathbf{Z} / 2\left[y_{1}, \ldots, y_{n}\right]^{G L_{n}} & =\operatorname{Im}\left[\operatorname{Res}_{1}: H^{*}\left(B \Sigma_{2^{n}}\right) \rightarrow H^{*}\left(B E^{n}\right)\right], \\
\mathbf{Z} / 2\left[y_{1}, \ldots, y_{n}\right]^{T_{n}} & =\operatorname{Im}\left[\operatorname{Res}_{2}: H^{*}\left(B \Sigma_{2^{n}, 2}\right) \rightarrow H^{*}\left(B E^{n}\right)\right] .
\end{aligned}
$$

Here $\Sigma_{m}$ denotes the symmetric group on $m$ letters, $\Sigma_{m, 2}$ is its Sylow 2subgroup, and $\operatorname{Res}_{1}, \operatorname{Res}_{2}$ are the restriction homomorphisms induced by the regular permutation representation $E^{n} \subset \Sigma_{2^{n}, 2} \subset \Sigma_{2^{n}}$ of $E^{n}$.

The mod 2 Steenrod algebra $A$ acts on $H^{*}\left(B E^{n}\right)=\mathbf{Z} / 2\left[y_{1}, \ldots, y_{n}\right]$ by means of the Cartan formula together with the relations

$$
S q^{0} y_{i}=y_{i}, \quad S q^{1} y_{i}=y_{i}^{2}, \quad S q^{j} y_{i}=0 \quad \text { for } 1<j \text { and } 1 \leq i \leq n .
$$

Since this action commutes with the actions of $G L_{n}$ and $T_{n}$, so it induces a natural action of $A$ on $\mathbf{Z} / 2\left[y_{1}, \ldots, y_{n}\right]^{G}$ for $G=G L_{n}$ or $T_{n}$. Note that, $\operatorname{deg} V_{n}=2^{n-1}$ and $\operatorname{deg} Q_{n, s}=2^{n}-2^{s}$ because $\operatorname{deg} y_{i}=1$.

In this paper the action of the Steenrod squares on the Dickson and Mùi invariants is explicitly determined by use of a very elementary method. We prove

\section{Theorem A.}

$$
S q^{i} V_{n}= \begin{cases}V_{n}, & i=0, \\ V_{n} Q_{n-1, s}, & i=2^{n-1}-2^{s}, 0 \leq s<n-1, \\ V_{n}^{2}, & i=2^{n-1}, \\ 0, & \text { otherwise. }\end{cases}
$$

We also have the following result, which was first obtained in joint work with Nguyên N. Hai [11] by another method.

Theorem B (Hai and Hung [11]).

$$
S q^{i} Q_{n, s}= \begin{cases}Q_{n, r}, & i=2^{s}-2^{r}, r \leq s, \\ Q_{n, r} Q_{n, t}, & i=2^{n}-2^{t}+2^{s}-2^{r}, r \leq s<t, \\ Q_{n, s}^{2}, & i=2^{n}-2^{s}, \\ 0, & \text { otherwise. }\end{cases}
$$

Many authors have studied the above action. However, their results can be divided into two kinds: Either they are only valid for $i$ a power of 2, or they 
are given only by inductive procedures. (See Singer [12] and Campbell [1] for results concerning Theorem A, and Madsen [5], Madsen-Milgram [6, Chapter 3], Smith-Switzer [14], and Wilkerson [16] for results related to Theorem B. See also May [9, §I.3], Mann [7] and Mann-Milgram [8] for results related to Theorem B with the coefficient ring $\mathbf{Z} / p$ for $p>2$.) Also by a direct computation, Singer obtained Theorem B for $n=2,3$ in [13].

It should be noted that the method which we present in this paper is very elementary. Roughly speaking, we read off the action of any Steenrod operation on the Dickson and Mùi invariants from the expansion of the Mùi invariants in terms of Dickson and Mùi invariants of fewer variables (see Lemmas 3.1 and 4.1 for details).

\section{Preliminaries}

Let $\xi_{i}$ be the Milnor element of dimension $2^{i}-1$ in the dual algebra $A_{*}$ of the mod 2 Steenrod algebra $A$. Milnor showed in [10] that

$$
A_{*} \cong \mathbf{Z} / 2\left[\xi_{1}, \xi_{2}, \ldots\right]
$$

as algebras.

Given a sequence of nonnegative integers $R=\left(r_{1}, \ldots, r_{m}\right)$, we denote by $S t^{R} \in A$ the dual of $\xi_{1}^{r_{1}} \cdots \xi_{m}^{r_{m}}$ with respect to the Milnor basis of $A_{*}$ consisting of all monomials in the $\xi_{i}$ 's. In particular, for $R=(r), S t^{(r)}=S q^{r}$. MadsenMilgram [6] and Mùi [4] have described $S t^{R}$ in terms of Dickson invariants by means of the homomorphism $d_{m}^{*} P_{m}$, a generalization of $d^{*} P$, which was first studied by Steenrod [15].

Suppose $X$ is a topological space. Let

$$
P_{m}: H^{i}(X) \rightarrow H^{2^{m} i}\left(E \Sigma_{2^{m}} \times_{\Sigma_{2^{m}}} X^{2^{m}}\right)
$$

be the Steenrod power map, which sends $u$ to $1 \otimes u^{2^{m}}$ at cochain level.

The inclusion $E^{m} \subset \Sigma_{2^{m}}$ mentioned in the introduction, together with the diagonal map of $X$ and the Künneth formula, induces the homomorphism

$$
d_{m}^{*}: H^{*}\left(E \Sigma_{2^{m}} \times_{\Sigma_{2^{m}}} X^{2^{m}}\right) \rightarrow H^{*}\left(B E^{m}\right) \otimes H^{*}(X) .
$$

As is well known [3], the Weyl group of $E^{m}$ in $\Sigma_{2^{m}}$ is isomorphic to $G L_{m}$. Under the identification $H^{*}\left(B E^{m}\right)=\mathbf{Z} / 2\left[x_{1}, \ldots, x_{m}\right]$, the Weyl group $G L_{m}$ acts on $\mathbf{Z} / 2\left[x_{1}, \ldots, x_{m}\right]$ as usual. A classical result asserts that

$$
\operatorname{Im}\left(d_{m}^{*}\right) \subset \mathbf{Z} / 2\left[x_{1}, \ldots, x_{m}\right]^{G L_{m}} \otimes H^{*}(X)
$$

Now $S t^{R}$ can be described as follows. 
2.1. Theorem (Madsen-Milgram [6, Chapter 3], Mùi [4]).

$$
d_{m}^{*} P_{m}(x)=\sum_{R=\left(r_{1}, \ldots, r_{m}\right)} Q_{m, 0}^{q-\left(r_{1}+\cdots+r_{m}\right)} Q_{m, 1}^{r_{1}} \cdots Q_{m, m-1}^{r_{m-1}} \otimes S t^{R}(x)
$$

for any $x \in H^{q}(X)$. Here $Q_{m, s}=Q_{m, s}\left(x_{1}, \ldots, x_{m}\right)$ for $0 \leq s<m$.

For $m=1, d_{1}^{*} P_{1}$ is nothing but $d^{*} P$ of Steenrod [15]. Mùi also observes that

2.2. Proposition [3, 4]. $d_{m}^{*} P_{m}$ is a natural homomorphism preserving cup product and satisfying

(i) $d_{m}^{*} P_{m}=d^{*} P d_{m-1}^{*} P_{m-1}^{*}$,

(ii) $d_{m}^{*} P_{m}(x)=V_{m+1}\left(x_{1}, \cdots, x_{m}, x\right)$.

Here $x$ is the generator of $H^{*}(B \mathbf{Z} / 2) \cong \mathbf{Z} / 2[x]$ with $\operatorname{deg} x=1$.

\section{Proof of Theorem A}

3.1. Lemma. There exists uniquely an expansion

$$
\begin{aligned}
& V_{m+n}\left(x_{1}, \ldots, x_{m}, y_{1}, \ldots, y_{n}\right) \\
& \quad=\sum_{R=\left(r_{1}, \ldots, r_{m}\right)} Q_{m, 0}^{2^{n-1}-\left(r_{1}+\cdots+r_{m}\right)} Q_{m, 1}^{r_{1}} \cdots Q_{m, m-1}^{r_{m-1}} \cdot \varphi^{R}\left(V_{1}, \ldots, V_{n}\right),
\end{aligned}
$$

with $\varphi^{R} \in \mathbf{Z} / 2\left[V_{1}, \ldots, V_{n}\right], Q_{m, s}=Q_{m, s}\left(x_{1}, \ldots, x_{m}\right)$ for $0 \leq s<m$, and $V_{r}=V_{r}\left(y_{1}, \ldots, y_{r}\right)$ for $1 \leq r \leq n$. Furthermore

$$
S t^{R}\left(V_{n}\right)=\varphi^{R}\left(V_{1}, \ldots, V_{n}\right) .
$$

Proof. From Proposition 2.2, we have

$$
d_{m}^{*} P_{m} V_{n}\left(y_{1}, \ldots, y_{n}\right)=V_{m+n}\left(x_{1}, \ldots, x_{m}, y_{1}, \ldots, y_{n}\right) .
$$

So, according to Theorem 2.1, there exists such an expansion. The uniqueness of the expansion follows from the algebraic independence of $V_{1}, \ldots, V_{n}$ over $\mathbf{Z} / 2\left[x_{1}, \ldots, x_{m}\right]$.

Finally, we obtain from another application of Theorem 2.1 the last equation of the lemma. 
3.2. Proof of Theorem A. We now apply Lemma 3.1 with $m=1$. Note that, in this case $S t^{(r)}$ is nothing but the Steenrod operation $S q^{r}$.

$$
\begin{aligned}
d^{*} P & V_{n}\left(y_{1}, \ldots, y_{n}\right)=V_{n+1}\left(x, y_{1}, \ldots, y_{n}\right) \\
& =\prod_{\lambda, \lambda_{i}}\left(\lambda x+\lambda_{1} y_{1}+\cdots+\lambda_{n-1} y_{n-1}+y_{n}\right) \\
& =\prod_{\lambda_{i}}\left(\lambda_{1} y_{1}+\cdots+\lambda_{n-1} y_{n-1}+y_{n}\right) \prod_{\lambda_{i}}\left(\lambda_{1} y_{1}+\cdots+\lambda_{n-1} y_{n-1}+x+y_{n}\right) \\
& =V_{n}\left(y_{1}, \ldots, y_{n}\right) V_{n}\left(y_{1}, \ldots, y_{n-1}, x+y_{n}\right) \\
& =V_{n}\left(y_{1}, \ldots, y_{n}\right)\left[\sum_{s=0}^{n-1} Q_{n-1, s}\left(y_{1}, \ldots, y_{n-1}\right)\left(x+y_{n}\right)^{2^{s}}\right] \\
& =V_{n}\left(y_{1}, \ldots, y_{n}\right)\left[V_{n}\left(y_{1}, \ldots, y_{n}\right)+\sum_{s=0}^{n-1} Q_{n-1, s}\left(y_{1}, \ldots, y_{n-1}\right) x^{2^{s}}\right] \\
& =V_{n}^{2}+\sum_{s=0}^{n-1} x^{2^{s}} \cdot V_{n} \cdot Q_{n-1, s} \cdot
\end{aligned}
$$

From this, Theorem A follows.

\section{Proof of Theorem B}

4.1. Lemma. There exists uniquely an expansion

$$
\begin{aligned}
& V_{m+n+1}\left(x_{1}, \ldots, x_{m}, y_{1}, \ldots, y_{n}, z\right) \\
& \quad=\sum_{s=0}^{n} P_{s}\left(x_{1}, \ldots, x_{m}, y_{1}, \ldots, y_{n}\right) V_{m+1}^{2^{s}}\left(x_{1}, \ldots, x_{m}, z\right)
\end{aligned}
$$

with $P_{s} \in \mathbf{Z} / 2\left[x_{1}, \ldots, x_{m}\right]^{G L_{m}} \otimes \mathbf{Z} / 2\left[y_{1}, \ldots, y_{n}\right]^{G L_{n}}$. Furthermore, if

$$
P_{s}=\sum_{R=\left(r_{1}, \ldots, r_{m}\right)} Q_{m, 0}^{2^{n}-2^{s}-\left(r_{1}+\cdots+r_{m}\right)} Q_{m, 1}^{r_{1}} \cdots Q_{m, m-1}^{r_{m-1}} \cdot \psi_{s}^{R}\left(Q_{n, 0}, \ldots, Q_{n, n-1}\right)
$$

with $Q_{m, r}=Q_{m, r}\left(x_{1}, \ldots, x_{m}\right), Q_{n, t}=Q_{n, t}\left(y_{1}, \ldots, y_{n}\right)$, then

$$
S t^{R}\left(Q_{n, s}\right)=\psi_{s}^{R}\left(Q_{n, 0}, \ldots, Q_{n, n-1}\right) \text {. }
$$

Proof. To prove the uniqueness of such an expansion, we suppose

$$
\sum_{s=0}^{n} F_{s}\left(x_{1}, \ldots, x_{m}, y_{1}, \ldots, y_{n}\right) V_{m+1}^{2^{s}}\left(x_{1}, \ldots, x_{m}, z\right)=0
$$

with the $F_{s}$ polynomials of the variables indicated.

We remark that $\operatorname{deg}_{z} V_{m+1}^{2^{s}}\left(x_{1}, \ldots, x_{m}, z\right)=2^{m+s}$. Observe the coefficient of the leading term $z^{2^{m+n}}$ on the left-hand side of the above equation and we have $F_{n}=0$. So, we get

$$
\sum_{s=0}^{n-1} F_{s} V_{m+1}^{2^{s}}=0
$$


Next, consider the leading term $z^{2^{m+n-1}}$ in the last equation, we obtain $F_{n-1}=$

0 . Continuing this argument, we get $F_{n}=F_{n-1}=\cdots=F_{0}=0$.

Now we prove the existence of such an expansion. Recall that

$$
V_{n+1}\left(y_{1}, \ldots, y_{n}, z\right)=\sum_{s=0}^{n} Q_{n, s}\left(y_{1}, \ldots, y_{n}\right) \cdot z^{2^{s}}
$$

(see Mùi [3, Appendix]). With Proposition 2.2 in hand, apply $d_{m}^{*} P_{m}$ to the two sides of this equality and obtain

$$
V_{m+n+1}\left(x_{1}, \ldots, x_{m}, y_{1}, \ldots, y_{n}, z\right)=\sum_{s=0}^{n} d_{m}^{*} P_{m}\left(Q_{n, s}\right) \cdot V_{m+1}^{2^{s}}\left(x_{1}, \ldots, x_{m}, z\right)
$$

This is the desired expansion with

$$
P_{s}=d_{m}^{*} P_{m}\left(Q_{n, s}\right) \in \mathbf{Z} / 2\left[x_{1}, \ldots, x_{m}\right]^{G L_{m}} \otimes \mathbf{Z} / 2\left[y_{1}, \ldots, y_{n}\right]^{G L_{n}}
$$

Moreover, according to Theorem 2.1, we have

$$
d_{m}^{*} P_{m} Q_{n, s}=\sum_{R=\left(r_{1}, \ldots, r_{m}\right)} Q_{m, 0}^{2^{n}-2^{s}-\left(r_{1}+\cdots+r_{m}\right)} Q_{m, 1}^{r_{1}} \cdots Q_{m, m-1}^{r_{m-1}} \cdot S t^{R}\left(Q_{n, s}\right)
$$

So we get the last conclusion of the lemma

$$
S t^{R}\left(Q_{n, s}\right)=\psi_{s}^{R}\left(Q_{n, 0}, \ldots, Q_{n, n-1}\right)
$$

4.2. Proof of Theorem B. Apply Lemma 4.1 with $m=1$ and remember that $S t^{(r)}=S q^{r}$. By the same argument as given in the proof of Theorem A, we have

$$
\begin{aligned}
V_{n+2}\left(x, y_{1}, \ldots, y_{n}, z\right)= & V_{n+1}\left(y_{1}, \ldots, y_{n}, z\right) V_{n+1}\left(y_{1}, \ldots, y_{n}, x+z\right) \\
= & V_{n+1}^{2}\left(y_{1}, \ldots, y_{n}, z\right) \\
& +V_{n+1}\left(y_{1}, \ldots, y_{n}, z\right) V_{n+1}\left(y_{1}, \ldots, y_{n}, x\right) \\
= & \sum_{s=0}^{n} Q_{n, s}^{2} z^{2^{s+1}}+\sum_{s=0}^{n} Q_{n, s^{s}} z^{s} \sum_{t=0}^{n} Q_{n, t} x^{t} \\
= & \sum_{s=0}^{n} Q_{n, s}^{2}\left(x^{2^{s}} z^{2^{s}}+z^{2^{s+1}}\right)+\sum_{r=0}^{n-1} Q_{n, r}\left(x^{2^{n}} z^{2^{r}}+x^{2^{r}} z^{2^{n}}\right) \\
& +\sum_{0 \leq r<t<n} Q_{n, r} Q_{n, t}\left(x^{2^{r}} z^{2^{t}}+x^{2^{t}} z^{2^{r}}\right) .
\end{aligned}
$$


Note that $V_{2}(x, z)=x z+z^{2}$. A simple computation leads us to

$$
\begin{aligned}
x^{2^{n}} z^{2^{r}}+x^{2^{r}} z^{2^{n}}= & x^{2^{n}-2^{r}}\left(x^{2^{r}} z^{2^{r}}+z^{2^{r+1}}\right)+x^{2^{n}-2^{r}-2^{r+1}}\left(x^{2^{r+1}} z^{2^{r+1}}+z^{2^{r+2}}\right)+\cdots \\
& +x^{2^{n}-2^{r}-\cdots-2^{n-1}}\left(x^{2^{n-1}} z^{2^{n-1}}+z^{2^{n}}\right) \\
= & \sum_{s=r}^{n-1} x^{2^{n}-\left(2^{s+1}-2^{r}\right)} V_{2}^{2^{s}}(x, z) \\
x^{2^{t}} z^{2^{r}}+x^{2^{r}} z^{2^{t}}= & x^{2^{t}-2^{r}}\left(x^{2^{r}} z^{2^{r}}+z^{2^{r+1}}\right)+\cdots \\
& +x^{2^{t}-2^{r}-\cdots-2^{t-1}}\left(x^{2^{t-1}} z^{2^{t-1}}+z^{2^{t}}\right) \\
= & \sum_{s=r}^{t-1} x^{2^{t}-\left(2^{s+1}-2^{r}\right)} V_{2}^{2^{s}}(x, z) .
\end{aligned}
$$

So we get

$$
\begin{aligned}
V_{n+2}\left(x, y_{1}, \ldots, y_{n}, z\right)= & \sum_{s=0}^{n} Q_{n, s}^{2} V_{2}^{2^{s}}+\sum_{r \leq s<n} x^{2^{n}-2^{s+1}+2^{r}} Q_{n, r} V_{2}^{2^{s}} \\
& +\sum_{r \leq s<t<n} x^{2^{t}-2^{s+1}+2^{r}} Q_{n, r} Q_{n, t} V_{2}^{2^{s}}
\end{aligned}
$$

From Lemma 4.1, this implies

$$
d^{*} P Q_{n, s}=Q_{n, s}^{2}+\sum_{r \leq s} x^{2^{n}-2^{s+1}+2^{r}} Q_{n, r}+\sum_{r \leq s<t} x^{2^{t}-2^{s+1}+2^{r}} Q_{n, r} Q_{n, t}
$$

Theorem B follows.

\section{ACKNOWLEDGMENT}

I would like to thank the Mathematical Sciences Research Institute, Berkeley, California, for the hospitality and the ideal working conditions while I was visiting there in the academic year 1989-1990.

Note added in proof. Recently, H. E. A. Campbell informed me that T. Bisson independently showed Theorem B in a private letter sent to Campbell.

\section{REFERENCES}

1. H. E. A. Campbell, Upper triangular invariants, Canad. Math. Bull. 28 (1985), 243-248.

2. L. E. Dickson, A fundamental system of invariants of the general modular linear group with a solution of the form problem, Trans. Amer. Math. Soc. 12 (1911), 75-98.

3. Huỳnh Mùi, Modular invariant theory and cohomology algebras of symmetric groups, J. Fac. Sci. Univ. Tokyo Sec. IA 22 (1975), 319-369.

4. __ Dickson invariants and Milnor basis of the Steenrod algebra, Eger Internat. Colloq. Topology (1983), 345-355.

5. I. Madsen, On the action of the Dyer-Lashof algebra in $H_{*}(G)$, Pacific J. Math. 60 (1975), 235-275. 
6. I. Madsen and R. J. Milgram, The classifying spaces for surgery and cobordism of manifolds, Ann. of Math. Studies, No. 92, Princeton Univ. Press, 1979.

7. B. M. Mann, The cohomology of the symmetric groups, Trans. Amer. Math. Soc. 242 (1978), 157-184.

8. B. M. Mann and R. J. Milgram, On the Chern classes of the regular representations of some finite groups, Proc. Edinburgh Math. Soc. 25 (1982), 259-268.

9. J. P. May, F. R. Cohen, and T. J. Lada, The homology of iterated loop spaces, Lecture Notes in Math., vol. 533, Springer-Verlag, Berlin and New York, 1976.

10. J. Milnor, The Steenrod algebra and its dual, Ann. of Math. 67 (1958), 150-171.

11. Nguyên N. Hai and Nguyên H. V. Hung, Steenrod operations on mod 2 homology of the iterated loop space, Acta Math. Vietnam. 13 (1988), 113-126.

12. W. M. Singer, On the construction of certain algebras over the Steenrod algebra, J. Pure Appl. Algebra 11 (1977), 53-59.

13. __ The transfer in homological algebra, Math. Z. 202 (1989), 493-523.

14. L. Smith and R. Switzer, Realizability and non-realizability of Dickson algebras as cohomology rings, Proc. Amer. Math. Soc. 89 (1983), 303-313.

15. N. E. Steenrod and D. B. A. Epstein, Cohomology operations, Ann. of Math. Studies, No. 50, Princeton Univ. Press, 1962.

16. C. Wilkerson, A primer on the Dickson invariants, Contemporary Mathematics, Amer. Math. Soc., Providence, R.I., vol. 19, 1983, pp. 421-434.

Mathematical Sciences Research Institute, 1000 Centennial Drive, Berkeley, CaliFORNIA 94720

Current address: Department of Mathematics, University of Hanôi, Dai hoc Tong hop, Hanôi, Viêtnam 